\title{
Emprendimiento opción para reactivar la economía y el desarrollo sostenible en comuna Santa Elena
}

\section{Entrepreneurship option to react the economy and sustainable development in comuna Santa Elena}

\author{
Magdalena Huilcapi Masacón $^{1}$ *, Julio Mora Aristega ${ }^{1}$ y Gabriela Castro López ${ }^{1}$ \\ 1 Universidad Técnica de Babahoyo \\ *mhuilcapi@utb.edu.ec
}

DOI: https://doi.org/10.26871/killkana_social.v2i3.324

\begin{abstract}
Resumen
El mundo globalizado nos expone día a día ante productos y servicios nuevos, la economía difícil de ciertos segmentos de la población, el auge que en los últimos tiempos ha tomado el concepto de emprendimiento, exige a las universidades el apoyo y el acompañamiento a los habitantes de las comunidades y sectores vulnerables, a fin de hacer realidad las ideas de emprendimiento como una opción para reactivar la economía del sector y, contribuir a enriquecer aspectos personales de los integrantes de la comunidad y mejorar las dinámicas en términos de desarrollo social, económico y ambiental, para ello fue necesario fomentar la generación de ideas de emprendimientos locales, asistirlos en el manejo de los recursos financieros y en la capacitación de personas para la puesta en marcha del negocio, que permita el impulso de la economía del sector; el papel activo en este proceso lo desempeñan los gobiernos locales, pues son ellos los catalizadores de las iniciativas de desarrollo económico local; La metodología utilizada fue de tipo cualitativa y cuantitativa, se aplicó el método inductivo y deductivo y análisis estadístico mediante el análisis de frecuencia y la aplicación de encuestas a las personas que viven en la comunidad de Santa Elena, el objetivo de este trabajo permitió identificar el aporte a la economía de las familias y de la sociedad que generan los emprendimientos, en función de que éste se ha constituido en una herramienta para la reactivación económica en beneficio del desarrollo sostenible del sector, llegando a la conclusión de que el apoyo a través de diferentes ejes es fundamental para que los emprendimientos generen bienestar, comodidad, fuentes de trabajo en el entorno familiar y social y que por ende mejoren las condiciones de vida.
\end{abstract}

Palabras clave: creatividad, calidad de productos, desarrollo local, emprendimiento, economía.

\begin{abstract}
The globalized world exposes us every day to new products and services, the difficult economy of certain segments of the population, the boom that in recent times has taken the concept of entrepreneurship, requires universities to support and support the inhabitants of communities and vulnerable sectors, in order to make the ideas of entrepreneurship a reality as an option to reactivate the economy of the sector and, contribute to enrich personal aspects of the members of the community and improve the dynamics in terms of social, economic and environmental, for this it was necessary to foment the generation of ideas of local enterprises, assist them in the management of financial resources and in the training of people for the start-up of the business, which allows the boost of the economy of the sector; the active role in this process is played by local governments, since they are the catalysts of local economic development initiatives; The methodology used was qualitative and quantitative, the inductive and deductive method was applied, and statistical analysis through the frequency analysis and the application of surveys to people living in the community of Santa Elena, the objective of this work was to identify the contribution to the economy of the families and of the society that generate the enterprises, in function of which this has constituted in a tool for the economic reactivation in benefit of the sustainable development of the sector, coming to the conclusion that the support through different axes is fundamental for the entrepreneurship to generate well-being, comfort, sources of work in the family and social environment and therefore improve living conditions.
\end{abstract}

Key words: creativity, product quality, local development, entrepreneurship, economics.

\section{Introducción}

Los cambios acelerados en el mundo de los negocios, causa dificultades a los emprendedores, uno de los más grandes problemas que enfrentan es lo difícil de perma- necer en el mercado, los inconvenientes que se presentan le hacen desistir, la imposibilidad de innovar para ser aceptado por los consumidores o usuarios, muchas veces por el desconocimiento, la falta de preparación académica, 
la adversidad económica o la situación cambiante de los mercados, se convierte en una barrera difícil de superar.

Los emprendimientos más exitosos corresponden a la línea de tecnologías, y se los califica así por la capacidad que tienen de retener personal con talentos, por la calidad de gestión y de productos, por ser innovadores y socialmente responsables (Huilcapi, Mora, y Escobar, 2017), se dice que los emprendimientos son la base de la economía de los pueblos, a nivel de las comunidades y de los sectores menos protegidos, éstos cobran mayor importancia.

El emprendimiento es un concepto nuevo relacionado con la economía, pues surge de la necesidad de las personas que, al enfrentarse a una difícil situación por acceder a un empleo, buscan la opción del emprendedurismo, como fuente para la generación de recursos económicos. Más tarde las ciencias humanas (sociología, psicología y la antropología) hacen su aporte al momento de analizar el comportamiento de las personas "como ser social y cómo el entorno influye directa o indirectamente en el ser humano" al considerar la alternativa del emprendimiento como el mecanismo para obtener ingresos, reactivar la economía y por ende mejorar sus condiciones y la calidad de vida; corresponde a la universidad desde la función vinculación "universidad - sociedad", brindar asesoramiento, acompañamiento, apoyo a las comunidades y sectores vulnerables, a fin de hacer realidad las ideas de emprendimiento que permitan mejorar las dinámicas en términos de desarrollo social, económico y ambiental, para que el desarrollo de las comunidades sea sostenible.

\section{Marco Teórico}

Se define al emprendimiento como el descubrimiento de oportunidades rentables (Shane S., 2000). (Roberts D y Woods, 2005) lo define como "la construcción, evaluación y persecución de oportunidades para el cambio social transformativo llevado a cabo por individuos visionarios y dedicados; en un artículo publicado por la (Durán, Fuenmayor, Cárdenas, y Hernández, 2016) se refiere al emprendimiento, como las competencias, habilidades y destrezas de los individuos para generar un proceso de negocio, de allí la importancia del rol de la universidad en cumplir con la función de vinculación con la sociedad para capacitar y apoyar en el desarrollo de los emprendimientos, a las personas de las comunidades, en vista de que para ellos ésta actividad se convierte en una alternativa para generar ingresos y mejorar las condiciones de vida de las personas; según (Durán y cols., 2016) mencionan lo que Paz, Prieto y García (2016), entienden por emprender, que "no es únicamente una oportunidad, sino una necesidad"; pues el emprendimiento no solo tiene sentido económico, sino que tiene connotación social debido a que se generan fuentes de empleo, y ésta es una condición que eleva el autoestima de las personas al sentirse útiles a la sociedad.

La mayoría de las definiciones coinciden en "la búsqueda de soluciones a problemáticas sociales que hay en las comunidades y que ven en el emprendimiento como una alternativa de solución"; entre los problemas sobresalen, la falta de oportunidades de empleo, la crisis económica y los problemas juveniles.

En este sentido es importante la socialización de experiencias emprendedoras de personas que tuvieron la oportunidad de ser exitosas con sus negocios, ellas sortearon una serie de barreras y dificultades que se les presentaron, pese a estas vicisitudes primaron en ellas los valores como la constancia, creatividad, perseverancia, asumir riesgo, responsabilidad, liderazgo espíritu de grupo, solidaridad, la capacidad de adaptación y la seguridad en sí mismo para poder triunfar.

\subsection{Pilares del emprendedor}

Además de los conocimientos, habilidades, valores que debe tener un emprendedor, se requieren de algunos pilares fundamentales relacionados con:

- Compromiso. - Cumplir con los acuerdos que realiza con otras personas.

- Responsabilidad. - Enfrentar las consecuencias de sus decisiones

- Aptitud. - Debe ser y estar preparado para iniciar su idea de negocio.

- Pasión. - Hacer sus actividades con gusto, disfrutar del trabajo, hacerlo con amor.

- Retorno de la Inversión (ROI). - Convertir rápidamente su idea en un proyecto rentable.

- Estrategia. - Desarrollar mente estratega es importante para crear y crecer un negocio, así como diseñar estrategias de mercadotecnia y comercialización.

- Capital. - Todo proyecto necesita el dinero para iniciar, pero además se requiere del capital intelectual, entre otros.

- Innovación. - La innovación es necesaria para crear e inventar nuevos productos y servicios, innovar los canales de distribución, las campañas publicitarias, innovar los sistemas de trabajo.

Según ("Diferencia entre emprender por necesidad y por oportunidad", 2018), hablar del emprendimiento como opción de vida para la generación de ingresos, es encontrar dos tipologías: los emprendimientos por oportunidad y por necesidad, según Rossnaira hay factores que identifican a cada uno de ellos:

El emprendimiento por necesidad, que es la solución a una necesidad propia o de su entorno que en algunos casos no está soportada por un estudio para entender el mercado y posibles clientes. O que su idea de negocio parte de uno tradicional y los componentes innovadores son pocos.

El emprendimiento por oportunidad, este modelo de negocio es escalable, es decir que se puede replicar en distintos lugares o sectores, y que, además está compuesto por equipos de trabajos con especialidades diferentes; para el caso de análisis los modelos de emprendimientos que han tenido éxito en otras comunidades, son los que se aspira que las personas puedan replicar en una de las comunidades de Santa Elena. 
En una entrevista realizada a varios emprendedores, Suleiman indica que existen cinco características para ser un emprendedor exitoso, según se muestra en el gráfico $\mathrm{N}^{\circ} 1$ , aunque la gran mayoría lo describe el emprendimiento como un trabajo difícil y desalentador (Suleiman, 2015).

Figura 1. Características del emprendedor

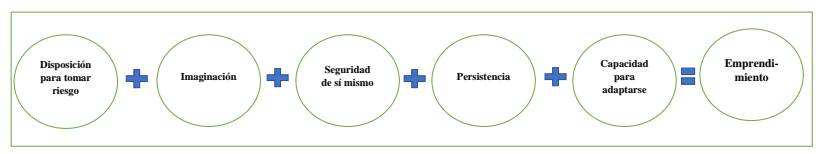

Fuente: (Suleiman, 2015)

Atendiendo a estas consideraciones el Estado Ecuatoriano a través de la Secretaría Nacional de Planificación y Desarrollo, (Secrertaría Técnica Plan Toda una Vida, 2017) en el objetivo número cinco, señala la importancia de "Impulsar la productividad y competitividad para el crecimiento económico sostenible de manera redistributiva y solidaria", en su política número seis, destaca el valor de "Promover la investigación, la formación, la capacitación, el desarrollo y la transferencia tecnológica, la innovación y el emprendimiento, la protección de la propiedad intelectual, para impulsar el cambio de la matriz productiva mediante la vinculación entre el sector público, productivo y las universidades", esto con la finalidad de que las áreas rurales, las comunidades puedan integrarse a la vida económica y productiva con valor agregado, generando los emprendimientos como elemento para ayudarse a la economía del hogar, a la empleabilidad, y al desarrollo, para mejorar los escenarios de vida de la familia.

En ese mismo sentido el objetivo cinco en su política número nueve señala que "Fortalecer y fomentar la asociatividad, los circuitos alternativos de comercialización, las cadenas productivas, negocios inclusivos y el comercio justo, priorizando la Economía Popular y Solidaria, para consolidar de manera redistributiva y solidaria la estructura productiva del país", (Secrertaría Técnica Plan Toda una Vida, 2017), es una condición más para que los emprendedores de las comunidades tengan otra alternativa para aportar a la economía e impulsar el desarrollo económico utilizando los recursos productivos del sector, manteniendo además la cultura e identidad de sus pueblos.

Considerando que el, emprendimiento sostenible es un fenómeno deseable, implementar políticas o programas de intervención social que favorezcan o potencialicen este tipo de actividad económica, es fundamental porque tiene como finalidad impulsar la economía y bienestar social (Chirinos, s.f.), en Ecuador el Plan Nacional de Desarrollo 2017-2021Toda una Vida, en su objetivo seis, promulga el desarrollo de las capacidades productivas y del entorno (...) entre las políticas para alcanzar el objetivo destaca en la numero uno, que es necesario "fomentar el trabajo y el empleo digno con énfasis en zonas rurales (...) fortaleciendo el apoyo focalizado del estado e impulsando el emprendimiento, (Secrertaría Técnica Plan Toda una Vida, 2017), sólo si a través de la Secretaría Técnica del Estado se logra el cumplimiento de éstos objetivos, el Estado Ecuatoriano está garantizando la sostenibilidad de los emprendimientos.

\subsection{Reactivación económica y el desarrollo sostenible}

Para (Pérez Peralta y Chirinos Araque, 2016), el emprendimiento sostenible se caracteriza por ser una forma de integración social, humana y económica, con la finalidad de realizar iniciativas de negocios que permitan el aprovechamiento de las oportunidades que se presentan en el entorno. Crear productos y servicios, que son necesarios para todos los niveles de la sociedad, es una forma muy eficaz de atacar la pobreza (Salinas, 2016).

Sin embargo, asumir el riesgo y gestionarlo es una característica del emprendedor, (...) el comportamiento humano es a veces impulsivo, ilógico, arriesgado y precipitado. ¿Qué es el riesgo? Es una probabilidad, una lotería al revés con castigo en vez de premio; una empresa tiene varios riesgos posibles. A menudo no totalmente inevitables pero al menos minimizables (Artal Castells, s.f.), todos estos aspectos afectan a los emprendedores, por lo que a veces al primer problema optan por salirse de la actividad.

\subsection{Desarrollo Sostenible}

Según (Quiroga Martínez, 2009) el desarrollo sostenible, implica la consideración simultánea de cuatro dimensiones constitutivas a saber: ambiental, económica, social e institucional, en el proceso de desarrollo; en la que destacan indicadores de dimensión económica, ambiental, de producción y manejo de residuos, dimensión social, nivel de educación, acceso a la salud y acceso a internet.

En este contexto para hablar de sostenibilidad se requiere de modelos actitudinales que hagan más viable y deseable la creación de emprendimientos sostenibles, se dice que los emprendimientos sociales son las empresas basadas en la comunidad de base común, que actúa corporativa y emprendedoramente en busca del bien común (López Puga, 2012).

\section{Metodología}

La metodología utilizada es de carácter cuantitativa, la misma que ha permitido detallar y analizar la problemática relacionada con los emprendimientos, así como la recolección de los datos cuantitativos a través de las encuestas realizadas, metodología que ha permitido identificar las características de la población objeto de estudio, se diseñó y aplicó un cuestionario de preguntas a 34 emprendedores de las 29 comunidades de Santa Elena. El tratamiento y análisis estadístico de la información contenida en la base de datos de la investigación realizada, se procesó con el paquete estadístico SPSS, v 22, debido a su capacidad de generación de informes y facilidad para importar datos desde Excel. 


\section{Resultados y Discusión}

\subsection{Motivación}

La Tabla $\mathrm{N}^{\circ} 1$ hace referencia a la motivación de las personas para iniciar un emprendimiento.

Tabla 1 Motivación para iniciar un emprendimiento

\begin{tabular}{|l|c|c|}
\hline \multicolumn{1}{|c|}{ Opciones } & Frecuencia & Porcentaje \\
\hline Es mi pasión & 12 & $35 \%$ \\
\hline Generar oportunidad de trabajo & 15 & $44 \%$ \\
\hline Involucrarse con la comunidad & 7 & $21 \%$ \\
\hline Total & 34 & $100 \%$ \\
\hline
\end{tabular}

Fuente: Encuesta realizada en las comunidades. 2018

Elaboración: Autores

Respecto a la motivación por iniciar un emprendimiento, el $44 \%$ de los encuestados expresó que lo hizo por generar una oportunidad de empleo, en tanto que el $35 \%$ lo hizo realmente por que la actividad le agrada. Las personas se motivan por la necesidad trabajar, por sentirse productivas y por integrarse a la sociedad.

\subsection{Financiamiento para iniciar su emprendimiento}

La tabla $\mathrm{N}^{\mathrm{a}} 2$ se refiere a si el emprendedor tiene financiamiento, se sabe que los recursos económicos generan estabilidad emocional y económica al emprendedor, contar con este recurso para iniciar es la clave.

Tabla 2 Financiamiento para iniciar emprendimiento

\begin{tabular}{|l|c|c|}
\hline \multicolumn{1}{|c|}{ Opciones } & Frecuencia & Porcentaje \\
\hline $\mathrm{Si}$ & 4 & $12 \%$ \\
\hline $\mathrm{No}$ & 30 & $88 \%$ \\
\hline Total & 34 & $100 \%$ \\
\hline
\end{tabular}

Fuente: Encuesta realizada en las comunidades. 2018 Elaboración: Autores

Respecto a contar o no con financiamiento para iniciar un negocio, el $88 \%$ de los encuestados respondió que no posee, sólo un $12 \%$ señaló que si tenía los recursos para iniciarse en la actividad; situación que muchas veces se convierte en una barrera para iniciar la actividad económica.

En la Tabla $\mathrm{N}^{\circ} 3$, se presentan los resultados relacionados con las fuentes para la obtención del financiamiento, aspecto que para algunos es una exigencia difícil de cumplir, razón por las que esto se considera una dificultad para el emprendedor.
Tabla 3 Obtener financiamiento

\begin{tabular}{|l|c|c|}
\hline \multicolumn{1}{|c|}{ Opciones } & Frecuencia & Porcentaje \\
\hline Muy difícil & 18 & $53 \%$ \\
\hline Difícil & 13 & $38 \%$ \\
\hline Nada difícil & 3 & $9 \%$ \\
\hline Desconozco & 0 & $0 \%$ \\
\hline Total & 34 & $100 \%$ \\
\hline
\end{tabular}

Fuente: Encuesta realizada en las comunidades. 2018

Elaboración: Autores

En las políticas de estado se consideran diversas formas de apoyo al emprendedor, sin embargo, por el desconocimiento muchas veces las personas no acceden y consideran que es muy difícil, así lo expresó el $53 \%$ de los encuestados, sólo un $9 \%$ dijo que no es nada difícil conseguir el financiamiento. El apoyo es muy importante para que el emprendedor inicie sus actividades, pues éste se convierte en una palanca sicológica y financiera que le ofrece seguridad.

La Tabla N 4 refiere las variadas formas mediante las cuales se puede apoyar al emprendedor, cada una de acuerdo a su condición, experiencia, actitud, y aptitud.

Tabla 4 Apoyo para iniciar un emprendimiento

\begin{tabular}{|l|c|c|}
\hline \multicolumn{1}{|c|}{ Opciones } & Frecuencia & Porcentaje \\
\hline Económico & 12 & $35 \%$ \\
\hline Moral & 6 & $18 \%$ \\
\hline Académico & 12 & $35 \%$ \\
\hline Otros & 4 & $12 \%$ \\
\hline Total & 34 & $100 \%$ \\
\hline
\end{tabular}

Fuente: Encuesta realizada en las comunidades. 2018 Elaboración: Autores

Respecto a la pregunta $\mathrm{N}^{\circ} 4$ relacionada con el apoyo que el emprendedor considera más importante, el $35 \%$ señaló que es el económico y en igual porcentaje el académico, pues le permite adquirir todos los insumos, materiales, herramientas y piezas necesarias para iniciar el negocio, y tomar las mejores decisiones respectivamente, un pequeño porcentaje estima que el apoyo moral es muy necesario.

La tabla $\mathrm{N}^{\circ} 5$, considera los negocios que se repiten, pues la escasa preparación académica no le permite generar ideas nuevas e innovadoras, y no les queda otra oportunidad que emprender con un negocio que ya existen, dañando así el mercado, y afectando al número de clientes que tienen dichos emprendimientos. 
Tabla 5 Emprendimientos en la comunidad

\begin{tabular}{|l|c|c|}
\hline \multicolumn{1}{|c|}{ Opciones } & Frecuencia & Porcentaje \\
\hline Alimentos & 9 & $26 \%$ \\
\hline Tecnología & 7 & $20 \%$ \\
\hline Bazares & 3 & $9 \%$ \\
\hline Librerías & 4 & $12 \%$ \\
\hline Belleza & 8 & $24 \%$ \\
\hline Industrializados & 3 & $9 \%$ \\
\hline Total & 34 & $100 \%$ \\
\hline
\end{tabular}

Fuente: Encuesta realizada en las comunidades. 2018

Elaboración: Autores

La tabla $\mathrm{N}^{\circ} 5$, destaca los emprendimientos que más predominan, y en la encuesta realizada se logró determinar que los alimentos son los negocios que más predominan con un $26 \%$, seguido de los negocios de belleza con un $24 \%$, un $20 \%$ se pronunció por la tecnología, hay poca variedad en los negocios, lo que evidencia la escasa generación de ideas innovadoras que revolucionen el mercado y vuelvan atractivo.

La tabla $\mathrm{N}^{\circ} 6$ hace referencia a la capacitación para iniciar con pie firme el negocio es clave, si las familias no están capacitadas, corresponde prepararse de forma autónoma para lograr el éxito.

Tabla 6 Entidades que dieron capacitación en emprendimiento

\begin{tabular}{|l|c|c|}
\hline \multicolumn{1}{|c|}{ Opciones } & Frecuencia & Porcentaje \\
\hline Universidades & 12 & $35 \%$ \\
\hline Organismos estatales & 12 & $35 \%$ \\
\hline Organismos no gubernamentales & 6 & $18 \%$ \\
\hline Otros & 4 & $12 \%$ \\
\hline Total & 34 & $100 \%$ \\
\hline
\end{tabular}

Fuente: Encuesta realizada en las comunidades. 2018

Elaboración: Autores

En la tabla $\mathrm{N}^{\circ} 6$, se analiza lo importante que es la capacitación, ya que ésta les permite actuar técnicamente en los emprendimientos, el $35 \%$ dice que recibe capacitación de las universidades, otro porcentaje igual expresó que los capacitan los entes estatales, demostrando que en parte la universidad está asumiendo el rol que le corresponde

La tabla $\mathrm{N}^{\circ} 7$ hace referencia a la generación de empleos como arista de la economía para generar bienestar es muy importante.

Tabla 7 El emprendimiento genera empleos

\begin{tabular}{|l|c|c|}
\hline \multicolumn{1}{|c|}{ Opciones } & Frecuencia & Porcentaje \\
\hline Fuentes de trabajo & 11 & $32 \%$ \\
\hline Reactivación económica & 13 & $39 \%$ \\
\hline Bienestar - comodidad & 10 & $29 \%$ \\
\hline Total & 34 & $100 \%$ \\
\hline
\end{tabular}

Fuente: Encuesta realizada en las comunidades. 2018

Elaboración: Autores

En ésta tabla se analiza lo expresado por los entrevistados cuando fueron consultados si el emprendimiento genera fuentes de trabajo, el $32 \%$ expresó que sí, el $39 \%$ considera que la economía se reactiva en base a los emprendimientos, en tanto que el $29 \%$ dice que genera bienestar y comodidad.

La variación es mínima respecto de la percepción que tienen los emprendedores, vale destacar que las tres alternativas, son elementos que aportan al cambio de la matriz productiva y al desarrollo de las comunidades.

La tabla $\mathrm{N}^{\circ} 8$ destaca algunos de los elementos que inciden en los emprendimientos, sin duda que la formación académica es considerada como uno de los componentes externos que coadyuvan al éxito de los emprendimientos.

Tabla 8 Elementos extrínsecos que inciden en emprendimientos exitosos

\begin{tabular}{|l|c|c|}
\hline \multicolumn{1}{|c|}{ Opciones } & Frecuencia & Porcentaje \\
\hline Financiamiento & 6 & $18 \%$ \\
\hline Incentivos gubernamentales & 2 & $6 \%$ \\
\hline Asesoramiento & 12 & $35 \%$ \\
\hline Formación académica & 14 & $41 \%$ \\
\hline Total & 46 & $100 \%$ \\
\hline
\end{tabular}

Fuente: Encuesta realizada en las comunidades. 2018

Elaboración: Autores

Respecto a la Tabla $\mathrm{N}^{\circ} 8$, relacionada con los elementos extrínsecos que inciden en el éxito de los negocios, el $41 \%$ de los encuestados manifestó que es la formación académica, un $35 \%$ dice que es el asesoramiento, en tanto que un $18 \%$ expresó que es el financiamiento; se estima que, si el emprendedor no posee formación académica, el asesoramiento es la clave para desarrollar sus emprendimientos y que este sea exitoso.

\section{Conclusiones y Recomendaciones}

Los emprendimientos tienen un solo objetivo, solucionar los problemas de tipo social; por lo que se orientan en buscar rentabilidad que permita mejorar las condiciones de vida de las personas; siempre contribuyendo a la formación e incremento de un colchón de seguridad, para enfrentar las dificultades en el futuro.

La actuación de emprendedores con las características descritas para que lleguen a ser exitosos, requiere de un proceso complejo, si no se cuenta con el apoyo de organismos estatales, de allí que, para la comunidad objeto de estudio, cobra relevancia el Plan de Desarrollo toda una Vida, que el gobierno pone en práctica.

La educación es otra arista necesaria para el desarrollo de los emprendimientos basados en economía sostenible y si a ésta le añade la experiencia en la actividad no será difícil alcanzar el éxito; sin embargo, eso no resta posibilidades a los noveles emprendedores para que ellos también logren ser exitosos en los negocios.

\section{Referencias Bibliográficas}

Artal Castells, M. (s.f.). Dinamización de las ventas: el proceso comercial. España: ESIC Editorial. 
Chirinos, Y. (s.f.). Emprendimiento Sostenible como política de Estado. VII Jornadas Nacionales y IV internacionales de investigación de la URBE, 14681482.

Diferencia entre emprender por necesidad y por oportunidad. (2018). Emprender, s/n.

Durán, S., Fuenmayor, A., Cárdenas, S., y Hernández, R. (2016). Emprendimiento como proceso de responsabilidad social en Instituciones de Educación Superior en Colombia y Venezuela. Desarrollo Gerencial Revista de la Facultad de Ciencias Económicas Administrativas y Contables, 58-75.

Huilcapi, M., Mora, J., y Escobar, D. (2017). Emprendimiento e innovación. aristas para reactivar la economia. Guaranda, Bolivar, Ecuador: UEB.

López Puga, J. (2012). Modelos actitudinales y emprendidmiento sostenible. Cuides, Universidad de Almeria, 111-131.

Pérez Peralta, C. M., y Chirinos Araque, Y. d. V. (2016). La Responsabilidad Social Universitaria: emprendimiento sostenible como impacto de intervención en comunidades vulnerables. Revista FAN, 91-110.

Quiroga Martínez, R. (2009). Guía metodológica para desarrollar indicadores ambientales y de desarrollo sostenible en paises de América Latina y el Caribe (Inf. Téc.). Chile.

Roberts D, y Woods, C. (2005). Cambios para priorizar la ciudadanía corporativa. Business Review de la
Universidad de Auckland, 45-51.

Salinas, R. B. (2016). Blog de Emprendimiento.

Descargado de http: / / www.ricardosalinas . com/blog/blog . aspx? GUID=cd59b247

$-2 \mathrm{~b} 5 \mathrm{f}-45 \mathrm{a} 0-864 \mathrm{e}-\mathrm{a} 122372 \mathrm{a} 45 \mathrm{c} 0$

Secrertaría Técnica Plan Toda una Vida. (2017).

Descargado de http://www.planificacion . gob.ec/wp-content/uploads/ downloads/2017/10/PNBV.pdf

Shane S., S. y. V. (2000). Emprendimiento Social: Revisión de literatura. Estudios Gerenciales, 217-226.

Suleiman, S. (2015). CNN. Descargado de http:// cnnespanol.cnn.com

Recibido: 30 de junio de 2018

Aceptado: 3 de septiembre de 2018 